\title{
ATM mutations improve radio-sensitivity in wild-type isocitrate dehydrogenase- associated high-grade glioma: retrospective analysis using next-generation sequencing data
}

Nalee Kim ${ }^{1,2}$, Se Hoon Kim ${ }^{3,4}$, Seok-Gu Kang 4,5, Ju Hyung Moon ${ }^{4,5}$, Jaeho Cho ${ }^{1,4}$, Chang-Ok Suh ${ }^{6}$, Hong In Yoon ${ }^{1,4^{*} \dagger}$ (I) and Jong Hee Chang ${ }^{4,5^{* \dagger}}$

\begin{abstract}
Background: To identify the association between somatic ataxia-telangiectasia mutated (ATM) mutations and improved radio-sensitivity, we retrospectively reviewed next-generation sequencing data from patients diagnosed with isocitrate dehydrogenase (IDH)-wildtype high-grade glioma.

Methods: We included 39 individuals with (IDH)-wildtype high-grade glioma (diffuse astrocytoma $n=2$, anaplastic astrocytoma $n=10$, and glioblastoma $n=27$ ) not subjected to gross tumor resection and undergoing radiation therapy with a median total dose of $60 \mathrm{~Gy}$ in 30 fractions. The mutational status of the ATM gene was obtained through next-generation sequencing using a TruSight Tumor 170 cancer panel. Disease progression was defined according to the Response Assessment in Neuro-Oncology (RANO) criteria as well as neurologic and clinical findings.

Results: Among the 39 samples, ATM mutations (ATM mut(+)) were detected in $26 \%$ of cases $(n=10)$. No significant differences were observed in the characteristics of the patients or tumors. Among the 10 patients in the ATM mut(+) group, there were 6 patients with glioblastoma and 4 patients with anaplastic astrocytoma. Most mutations were missense mutations $(n=8,80 \%)$. With a median follow-up of 16.5 mo (interquartile range, 11.4-19.8), ATM mut(+) exhibited 1-year in-field control of 100\% compared with $44.1 \%$ in the ATM mut(-) group $(p=0.002)$. There was no difference in the out-field control rate or overall survival between the two groups $(p=0.861$ and $p=0.247$, respectively).
\end{abstract}

(Continued on next page)

\footnotetext{
*Correspondence: yhi0225@yuhs.ac; changjh@yuhs.ac

${ }^{\dagger}$ Hong In Yoon and Jong Hee Chang contributed equally to this work. 'Department of Radiation Oncology, Yonsei Cancer Center, Severance Hospital, Yonsei University Health System, Yonsei University College of Medicine, 50-1 Yonsei-ro, Seodaemun-gu, Seoul 03722, Republic of Korea

${ }^{4}$ Brain Tumor Center, Severance Hospital, Yonsei University Health System,

Yonsei University College of Medicine, 50-1 Yonsei-ro, Seodaemun-gu, Seoul 03722, Republic of Korea

Full list of author information is available at the end of the article
}

(C) The Author(s). 2020 Open Access This article is licensed under a Creative Commons Attribution 4.0 International License, which permits use, sharing, adaptation, distribution and reproduction in any medium or format, as long as you give appropriate credit to the original author(s) and the source, provide a link to the Creative Commons licence, and indicate if changes were made. The images or other third party material in this article are included in the article's Creative Commons licence, unless indicated otherwise in a credit line to the material. If material is not included in the article's Creative Commons licence and your intended use is not permitted by statutory regulation or exceeds the permitted use, you will need to obtain permission directly from the copyright holder. To view a copy of this licence, visit http://creativecommons.org/licenses/by/4.0/. The Creative Commons Public Domain Dedication waiver (http://creativecommons.org/publicdomain/zero/1.0/) applies to the data made available in this article, unless otherwise stated in a credit line to the data. 
(Continued from previous page)

Conclusions: Our results demonstrated that ATM mutations might be involved in the increased radio-sensitivity with excellent in-field control despite the aggressive nature of IDH-wildtype high-grade glioma. Further studies are necessary to uncover the potential role of ATM as a biomarker and candidate therapeutic target in high-grade gliomas.

Keywords: ATM, IDH-wild type high-grade glioma, Radiosensitivity, Next-generation sequencing, Radiation therapy

\section{Background}

Isocitrate dehydrogenase (IDH)-wildtype high-grade glioma (diffuse astrocytoma, anaplastic astrocytoma, and glioblastoma multiforme $[\mathrm{GBM}]$ ), being an aggressive brain tumor, has been correlated with poor prognosis despite the best trimodality treatment approaches (surgery, chemotherapy, and radiation therapy $(\mathrm{RT})$ ). This poor prognosis has been attributed to the intrinsic radio- and chemo-resistance of the tumor [1]. Although there are several clinical and molecular factors known to be prognostic factors for high-grade glioma, including methylation of O-6methylguanine-DNA methyltransferase (MGMT) promoter, age, involvement of the subventricular zone (SVZ), extent of resection, and sex [2], most patients are treated with a uniform adjuvant treatment approach (i.e., one-size-fits-all).

The ataxia-telangiectasia mutated (ATM) gene encodes a serine/threonine protein kinase known to be activated by autophosphorylation upon DNA double-strand breaks arising from ionizing radiation. The prevalence of ATM mutations $(A T M$ mut $(+))$ in high-grade glioma is known to be less than $5 \%$ [3]; hence, owing to this rarity, there have been no clinical reports on such patients. Similar to the approach employing a PARP inhibitor targeting BRCA1 mutations in breast and ovarian cancers [4], several preclinical studies on the inhibition of ATM have been performed to enhance radio-sensitivity in other solid tumors $[5,6]$ and high-grade gliomas [7-10]. Rainey et al. discovered that transient inhibition of ATM by chemicals (CP466722) sensitizes HeLa cells and cells expressing $B C R-A b l$ to ionizing radiation [6]. Recently, Golding et al. demonstrated that dynamic ATM inhibition with sub-micromolar concentrations of KU-60019 slightly increased RT-induced cell killing in human glioblastoma cells [8]. These studies intrigued many physicians to identify the clinical significance of ATM mut(+) in real practice.

With the emergence of state-of-the-art sequencing, next-generation sequencing (NGS) [11], physicians have easy access to the ATM mutational status of every patient. In this context, we undertook a retrospective analysis of NGS data to address the radio-sensitivity of ATM mut(+) and its impact on clinical outcomes in patients with IDH-wildtype high-grade glioma.

\section{Methods}

Patient selection

As NGS data for gliomas have been utilized in our institution from 2017, patients with newly diagnosed WHO grade II with IDH-wildtype, WHO grade III and IV type gliomas, according to the new 2016 WHO classification, were screened between June 2017 and December 2018. Accordingly, we screened for patients treated with RT following surgery and patients with available NGS data $(n=144)$. Patients were excluded from the study if one of the following criteria was met: (1) they had undergone gross total removal of the tumor, and thus it was not possible to investigate the response of the residual tumor to RT $(n=66)$; (2) they had been previously diagnosed with a primary CNS tumor $(n=15)$; (3) they had tumors with IDH mutations $(n=9)$; (4) they had undergone hypofractionated RT $(n=2)$; (5) peritumoral edema was not included in RT fields $(n=5)$; (6) they could not provide follow-up images $(n=5)$; and (7) they could not complete RT $(n=3)$. Finally, 39 patients, including 10 patients with $A T M$ mutations (ATM mut(+) group) and 29 patients without ATM mutations (ATM mut(-) group), were included in our cohort. The study protocol was approved by the institutional review board (No.42019-0009), and the requirement for the provision of informed consent was waived because of the retrospective nature of this study.

\section{Multi-modal treatments}

Treatments and follow-up for every patient were performed by a multidisciplinary neuro-oncology board, including neurosurgeons, radiation oncologists, neuroradiologists, neuropathologists, and medical oncologists [2]. All patients were evaluated based on perioperative and follow-up magnetic resonance images (MRI) and clinical symptoms. The involvement of the SVZ was assessed via preoperative MRI according to a standardized spatial classification [12]. Navigation-guided surgery following the maximal safe resection protocol was performed for all patients, except for those who underwent stereotactic biopsy. The extent of surgery was evaluated using immediate postoperative gadolinium-enhanced T1-weighted MRI obtained within $48 \mathrm{~h}$ after surgery and then categorized as total (absence of visible contrastenhanced portion), subtotal (at least $90 \%$ of the tumor 
removed), partial (less than $90 \%$ of the tumor removed), or biopsy (in case of stereotactic biopsy) [13].

Conventional fractionated RT with a median total dose of $60 \mathrm{~Gy}$ (interquartile range, IQR: 60.0-60.0) was applied in 30 fractions to the gross tumor volume. With postoperative MRI, both the resection cavity and residual tumor were included in the gross tumor volume. The clinical target volume was delineated to include the peritumoral edema with a 1 - or $1.5-\mathrm{cm}$ margin on T2weighted fluid-attenuated inversion recovery postoperative MRI, and a median total dose of $49.5 \mathrm{~Gy}$ (IQR: $48.0-51.0)$ in 30 fractions was then applied to the clinical target volume [14, 15]. Following the treatment strategy followed at our institution, there was no difference in the dose prescription of the protocol according to pathology: all patients were treated with intensitymodulated RT using Tomotherapy (Hi-Art TomoTherapy; Accuray, Sunnyvale, CA, USA).

In the case of patients with GBM, during RT, all patients concomitantly underwent a daily administration of temozolomide $\left(75 \mathrm{mg} / \mathrm{m}^{2}\right.$ of body surface area per day, 7 days per week, from the first to the last day of RT), followed by the administration of adjuvant temozolomide $\left(150-200 \mathrm{mg} / \mathrm{m}^{2}\right.$ for 5 days during each 28 -day cycle). Regarding patients with anaplastic astrocytoma and WHO grade II with IDH-wildtype, 6 cycles of adjuvant procarbazine, lomustine, and vincristine (oral lomustine; $110 \mathrm{mg} / \mathrm{m}^{2}$ on day 1; oral procarbazine: 60 $\mathrm{mg} / \mathrm{m}^{2}$ per $\mathrm{d}$ from day 8 to day 21 ; and intravenous vincristine: $1.4 \mathrm{mg} / \mathrm{m}^{2}$ on day 8 and 29) were administered every 6 weeks for 9 months.

\section{Molecular analysis}

Representative formalin-fixed, paraffin-embedded tissues were analyzed by targeted NGS using the commercially available TruSight Tumor 170 panel (Illumina, Inc., San Diego, CA, USA). Detailed methods for the procedure of sequencing and data analysis have been previously described $[16,17]$. In the obtained NGS data, along with ATM mutations, we identified other frequent mutations found in gliomas, such as mutations in the Breast Cancer susceptibility gene1/2 (BRCA1/2), phosphatase and tensin homolog (PTEN), telomerase reverse transcriptase (TERT), and tumor protein p53 (TP53) genes. The DNA methylation status of the MGMT promoter was also examined [18]. Additionally, we stratified patients based on the consensus guidelines from the Consortium to Inform Molecular and Practical Approaches to CNS Tumor Taxonomy (cIMPACT-NOW) for the identification of gliomas appearing histologically as WHO grade II or III with molecular features of GBM using the following criteria: (1) amplification of epidermal growth factor receptor $(E G F R) \quad(n=1)$, (2) combined whole chromosome 7 gain and whole chromosome 10 loss, or (3) mutation of the TERT promoter $(n=5)$ [19].

\section{Follow-up}

Follow-up of all patients was performed until death or time of analysis. Most patients underwent MRI 1 month after the planned RT as well as every 3 months for the first 2 years, and every 6 to 12 months thereafter according to institutional policy. Disease progression was defined using the Response Assessment in NeuroOncology (RANO) criteria [20] as well as neurologic and clinical findings. All patients suspected of having progressive disease were determined as exhibiting progressive disease after discussion and joint decision made by the multidisciplinary team. Either in-field or out-field failure was defined based on the relationship between the RT field (within the region for $95 \%$ of the prescribed dose) and the volume of the recurrence tumor assessed by MRI .

\section{Statistical analysis}

Parsons' Chi-square or Fisher's exact test were used to analyze categorical variables, whereas the MannWhitney $U$ test (non-normally distributed data) was used to compare continuous variables when comparing differences in the characteristics of patients and treatments between the two groups. All events (including infield failure, out-field failure, and death) were measured from the day of diagnosis (day of surgery) to the time of the event. The Kaplan-Meier method was performed to estimate the in-field, out-field, and overall survival (OS) rates with the log-rank test used to assess prognostic significance. Univariable analyses of in-field and out-field control as well as OS were performed using Cox regression analysis. Further multivariable analysis was not performed because there was no statistically significant factor identified in the univariable analysis. A $p$-value < 0.05 was considered statistically significant. Statistical analyses were performed using SPSS (version 25.0.0; IBM Corp., Armonk, NY, USA) and R (version 3.6.2; R Foundation for Statistical Computing, Vienna, Austria; https://www.R-project.org/) software.

\section{Results}

The median age at diagnosis was 59 years (IQR: 48-63), and most patients presented with a Karnofsky performance scale (KPS) score $\leq 80$. Perioperative MRI revealed that $87.2 \%$ of tumors were located in the SVZ, and $48.7 \%$ of patients underwent subtotal removal. Methylation of the MGMT promoter was observed in $61.5 \%$ of patients. There was no significant difference in the characteristics of the patients and treatments between the ATM mut(+) and ATM mut(-) groups (Table 1). In addition, there was no difference in the frequency of 
Table 1 Patient and treatment characteristics

\begin{tabular}{|c|c|c|c|c|}
\hline & Total & ATM mut(-) & ATM mut(+) & $p$-value \\
\hline & $N=39$ & $N=29$ & $N=10$ & \\
\hline Median age $[\mathrm{QQR}]$, years & $59.0[47.5 ; 63.0]$ & $60.0[52.0 ; 64.0]$ & $49.0[37.0 ; 59.0]$ & 0.071 \\
\hline$<60$ years, $n(\%)$ & $22(56.4)$ & $14(48.3)$ & $8(80.0)$ & 0.169 \\
\hline$\geq 60$ years, $n(\%)$ & $17(43.6)$ & $15(51.7)$ & $2(20.0)$ & \\
\hline Sex, $n(\%)$ & & & & 0.727 \\
\hline Male & $20(51.3)$ & $14(48.3)$ & $6(60.0)$ & \\
\hline Female & $19(48.7)$ & $15(51.7)$ & $4(40.0)$ & \\
\hline Preoperative KPS, $n(\%)$ & & & & 0.580 \\
\hline$\leq 80$ & $28(71.8)$ & $22(75.9)$ & $6(60.0)$ & \\
\hline $90-100$ & $11(28.2)$ & $7(24.1)$ & $4(40.0)$ & \\
\hline Subventricular zone, $n$ (\%) & & & & 1.000 \\
\hline Free & $5(12.8)$ & $4(13.8)$ & $1(10.0)$ & \\
\hline Involvement & $34(87.2)$ & $25(86.2)$ & $9(90.0)$ & \\
\hline Gliomatosis, $n(\%)$ & & & & 0.515 \\
\hline No & $21(53.8)$ & $17(58.6)$ & $4(40.0)$ & \\
\hline Yes & $18(46.2)$ & $12(41.4)$ & $6(60.0)$ & \\
\hline Pathology*, n (\%) & & & & 0.380 \\
\hline Diffuse astrocytoma & $2(5.1)$ & $2(6.9)$ & $0(0.0)$ & \\
\hline Anaplastic astrocytoma & $10(25.7)$ & $6(20.7)$ & $4(40.0)$ & \\
\hline Glioblastoma & $27(69.2)$ & $21(72.4)$ & $6(60.0)$ & \\
\hline MGMT promoter, $n(\%)$ & & & & 1.000 \\
\hline Unmethylated & $15(38.5)$ & $11(37.9)$ & $4(40.0)$ & \\
\hline Methylated & $24(61.5)$ & $18(62.1)$ & $6(60.0)$ & \\
\hline Median Ki67 index [IQR], \% & $15.0[6.5 ; 26.2]$ & $15.0[6.5 ; 27.5]$ & $17.5[7.5 ; 30.0]$ & 0.617 \\
\hline$<15 \%, n(\%)$ & $16(41.0)$ & $12(41.4)$ & $4(40.0)$ & 1.000 \\
\hline$\geq 15 \%, n(\%)$ & $23(59.0)$ & $17(58.6)$ & $6(60.0)$ & \\
\hline Extent of resection, $n(\%)$ & & & & 0.562 \\
\hline Biopsy & $2(5.1)$ & $2(6.9)$ & $0(0.0)$ & \\
\hline Partial removal & $18(46.2)$ & $14(48.3)$ & $4(40.0)$ & \\
\hline Subtotal removal & $19(48.7)$ & $13(44.8)$ & $6(60.0)$ & \\
\hline Median total RT dose $[\mathrm{IQR}]$, Gy & $60.0[60.0 ; 60.0]$ & $60.0[60.0 ; 60.0]$ & $60.0[60.0 ; 60.0]$ & 0.600 \\
\hline Median total RT fractions [IQR], fx & $30.0[30.0 ; 30.0]$ & $30.0[30.0 ; 30.0]$ & $30.0[30.0 ; 30.0]$ & 0.631 \\
\hline
\end{tabular}

mutations in other genes (Additional file 1). Detailed information on each patient with an ATM mutation is listed in Table 2. Missense mutations (8 patients, $80 \%$ ) were the most common mutations in the ATM mut(+) group. There were six patients diagnosed with GBM and four patients with IDH-wildtype anaplastic astrocytoma. Based on preoperative MRI, the involvement of the SVZ (9 patients, 90.0\%) and the presence of gliomatosis (6 patients, $60.0 \%$ ) were frequently observed in the ATM $\operatorname{mut}(+)$ group.
The median follow-up for all patients was 16.5 mo (IQR: 11.4-19.8), and there was no difference in the follow-up period between the two groups (ATM mut(+): 18.0 mo (IQR: 11.4-20.8); ATM mut(-): 15.5 mo (IQR: 10.0-19.2), $\quad p=0.645)$. Patients with ATM mut(+) showed no in-field failure compared with the ATM mut(-) group (1-y in-field control rate: $100.0 \%$ vs. $44.1 \%$, $p=0.002$, Fig. 1a). Conversely, there was no difference in the out-field failure between the two groups (1-y outfield control rate: $71.4 \%$ vs. $51.9 \%, p=0.861$, Fig. $1 \mathrm{~b})$. Of 
Table 2 Detailed information on patients harboring the ATM mutation

\begin{tabular}{|c|c|c|c|c|c|c|c|c|c|c|}
\hline Patient number & $\# 1$ & \#2 & \#3 & $\# 4$ & \#5 & $\# 6$ & \#7 & $\# 8$ & $\# 9$ & $\# 10$ \\
\hline Mutation & $\begin{array}{l}\text { Missense } \\
\text { mutation }\end{array}$ & $\begin{array}{l}\text { Missense } \\
\text { mutation }\end{array}$ & $\begin{array}{l}\text { Missense } \\
\text { mutation }\end{array}$ & $\begin{array}{l}\text { Missense } \\
\text { mutation }\end{array}$ & $\begin{array}{l}\text { Missense } \\
\text { mutation }\end{array}$ & $\begin{array}{l}\text { Frameshift } \\
\text { deletion }\end{array}$ & $\begin{array}{l}\text { Missense } \\
\text { mutation }\end{array}$ & $\begin{array}{l}\text { Missense } \\
\text { mutation }\end{array}$ & $\begin{array}{l}\text { Frameshift } \\
\text { insertion }\end{array}$ & $\begin{array}{l}\text { Missense } \\
\text { mutation }\end{array}$ \\
\hline VAF (\%) & 43.01 & 53.22 & 46.3 & 79.92 & 11.8 & 25.88 & 92.49 & 6.45 & 5.08 & 0.369 \\
\hline Amino acid change & p.R2832H & p.R9240 & p.K92Т & p.P2974L & p.L822S & p.L2946Nfs*9 & p.К92T & p.L413| & p.S28212Vfs*3 & p.P260T \\
\hline Sequence change & c. $8495 \mathrm{G}>\mathrm{A}$ & c. $2771 \mathrm{G}>\mathrm{A}$ & c. $275 \mathrm{~A}>\mathrm{C}$ & c.8921C > T & c. $2465 \mathrm{~T}>\mathrm{C}$ & c.8835_8836delGT & c. $275 \mathrm{~A}>\mathrm{C}$ & c. $1237 C>A$ & c.8432dupA & c. $778 \mathrm{C}>\mathrm{A}$ \\
\hline Age, years & 63 & 51 & 47 & 35 & 32 & 43 & 37 & 58 & 63 & 59 \\
\hline Sex & Male & Female & Female & Female & Male & Male & Female & Male & Male & Male \\
\hline KPS & 70 & 90 & 80 & 90 & 80 & 80 & 90 & 80 & 100 & 60 \\
\hline Pathology & GBM & GBM & GBM & GBM & GBM & GBM & $\mathrm{AA}, \mathrm{IDH}-\mathrm{WT}$ & AA, IDH-WT & AA, IDH-WT & AA, IDH-WT \\
\hline $\begin{array}{l}\text { MGMT promoter } \\
\text { methylation }\end{array}$ & $(-)$ & $(+)$ & $(+)$ & $(-)$ & $(+)$ & $(+)$ & $(-)$ & $(-)$ & $(+)$ & $(+)$ \\
\hline SVZ involvement & Yes & None & Yes & Yes & Yes & Yes & Yes & Yes & Yes & Yes \\
\hline Gliomatosis & No & No & Yes & Yes & No & Yes & No & Yes & Yes & Yes \\
\hline Extent of resection & Subtotal & Subtotal & Partial & Subtotal & Subtotal & Subtotal & Partial & Subtotal & Partial & Subtotal \\
\hline Total RT dose, Gy & 60 & 60 & 60.2 & 60 & 60 & 60 & 60 & 60.2 & 60 & 60 \\
\hline PD & Yes & Yes & No & Yes & No & No & No & Yes & No & No \\
\hline PD interval, months & 11.6 & 18.9 & 18.0 & 14.4 & & & & 15.4 & & \\
\hline Progression site ${ }^{*}$ & Out-field & Out-field & Out-field & Out-field & & & & Out-field & & \\
\hline Salvage treatment & $C T x$ & Re-RT & BSC & BSC & & & & Re-RT & & \\
\hline Follow-up, months & 22.7 & 19.8 & 20.8 & 15.0 & 8.1 & 11.4 & 18.7 & 17.3 & 19.2 & 11.1 \\
\hline Survival & Dead & Alive & Dead & Dead & Alive & Alive & Alive & Alive & Alive & Alive \\
\hline
\end{tabular}

Abbreviations: VAF Variant Allele Frequency, KPS Karnofsky performance status, GBM glioblastoma, $A A$ anaplastic astrocytoma, IDH isocitrate dehydrogenase, WT wild-type, MGMT O[6]-methylguanine-DNA methyltransferase, SVZ subventricular zone, $R T$ radiation therapy, $P D$ progressive disease, $C T x$ chemotherapy, Re- $R T$ reirradiation, $B S C$ best supportive care

* Progression site was defined based on relationship between radiation field and recurrence site

23 out-field failures, 6 failures $(60.0 \%$ of $A T M$ mut(+)) occurred in the $A T M$ mut $(+)$ group and $17(58.6 \%$ of $A T M$ mut(-)) in the ATM mut(-) group. In addition, out-field failures in the ATM mut(+) group were observed to have developed at a median of 15.4 mo (IQR: 11.6-18.9) after diagnosis. In addition, 3 patients died of progressive disease in the ATM mut(+) group with a 2-y OS rate of $32.1 \%$, which was comparable to that of the ATM mut(-) group (39.9\%, $p=0.247$, Fig. 1c). Using
Cox regression univariable analysis, we noted that only the BRCA mutation status was associated with worse out-field control (Hazard Ratio, 2.44) and poor OS (Hazard Ratio, 2.78, Table 3).

In subsequent analysis employing the recommended diagnostic criteria for the molecular features of glioblastoma according to cIMPACT-NOW $(n=33), A T M$ $\operatorname{mut}(+)(n=6)$ was shown to be associated with better in-field control than ATM mut(-) $(n=27)$ (1-y in-field

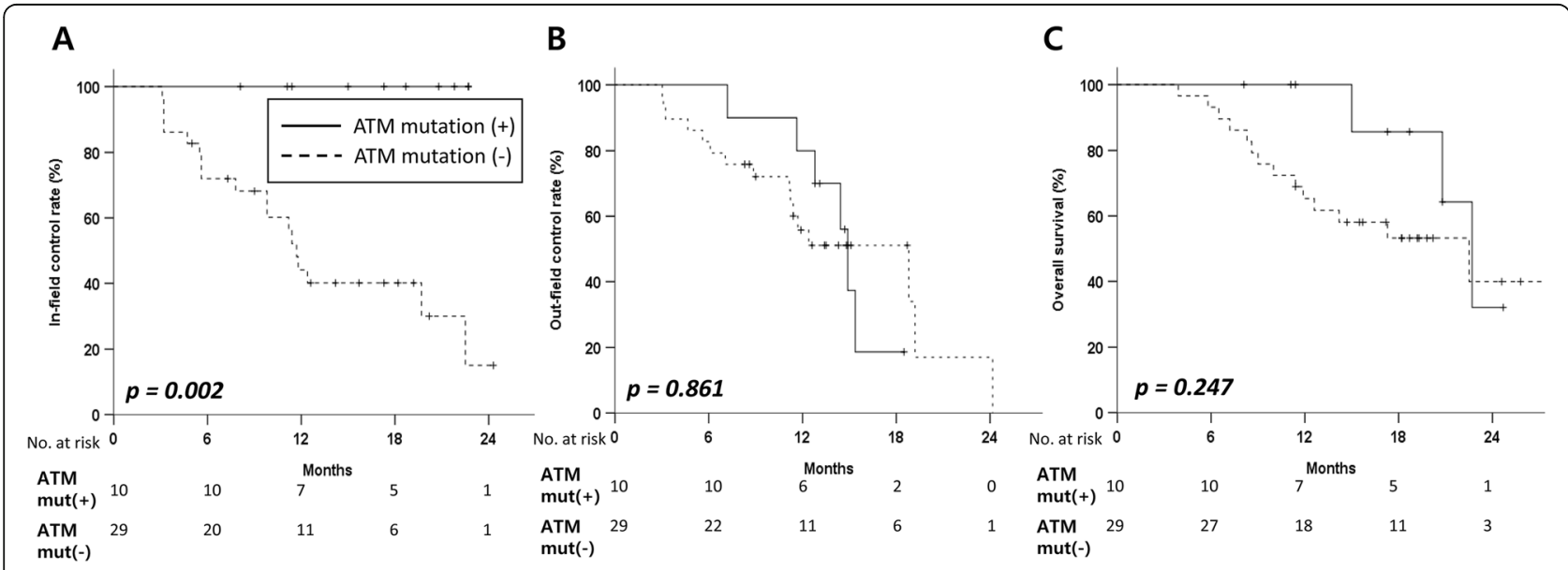

Fig. $1 \mathrm{ln}$-field $\mathbf{a}$ and out-field $\mathbf{b}$ control rates, and overall survival $\mathbf{c}$ of patients according to the mutational status of ATM 
control rate: $100.0 \%$ vs. $47.5 \%, p=0.021$; Fig. 2a, Additional File 2). The out-field control rate and OS values were comparable between the ATM mut(+) and ATM mut(-) groups in tumors with the molecular features of glioblastoma (Fig. 2b-c, Additional File 2). Even in tumors in contact with SVZ, the ATM mut(+) group was observed to be significantly associated with better in-field control than ATM mut(-) (1-y in-field control rate: $100.0 \%$ vs. $48.1 \%, p=0.003$, Additional File 3). In addition, there were no differences in the out-field control rate and OS values in tumors involving the SVZ $(p=0.711, p=0.433$, respectively, Additional File 3).

As the status of BRCA mutations was revealed to function as a prognostic factor for out-field control and OS, we performed further subgroup analysis stratified by the mutational status of $A T M$ and $B R C A$. Accordingly, patients with ATM mut(-) and BRCA mut(+) $(n=5)$ showed the worst clinical outcomes, with a 1-y in-field control rate of $20.0 \%$, out-field control rate of $20.0 \%$, and OS of $20.0 \%$ (Fig. 3a-c). In contrast, patients with ATM mut(+) and BRCA mut(-) $(n=7)$ were observed to exhibit statistically better clinical outcomes, with a $1-y$ in-field control rate of $100.0 \%$ and $2-y$ OS rate of $40.0 \%$ $(p=0.002$, and $p=0.031$, respectively). Patients with $A T M$ mut(+) and BRCA mut(+) $(n=3)$ showed comparable results to the ATM mut(+) and BRCA mut(-) group (1-y in-field control rate and 1-y OS rate of $100.0 \%$; $p=0.809$ for OS).

\section{Discussion}

We investigated the clinical impact of ATM mutations in patients with intracranial IDH-wildtype high-grade glioma undergoing RT following incomplete tumor resection. Interestingly, although well-known poor prognostic factors, such as either the presence of gliomatosis or the involvement of the SVZ, were predominant in the ATM mut(+) group, we observed excellent in-field control without in-field failure. However, it was shown that the ATM mut(+) group had no impact on the out-field area, demonstrating comparable out-field failures to the ATM mut(-) group. Hence, these clinical results illustrated the increased radio-sensitivity of tumors harboring somatic $A T M$ mutations.

There has been considerable accumulated preclinical evidence that $A T M$ might play a key role in the response to ionizing radiation. Preclinical studies on cell lines from patients with ataxia-telangiectasia syndromes have demonstrated that these cells exhibit increased sensitivity to radiation [21, 22]. Similarly, experimental inhibition of ATM was reported to influence cellular hypersensitivity to radiation $[5,6]$. This salutary effect on the radio-sensitivity of cells following the inhibition of $A T M$ was recently observed in GBM cell lines [7, 8], xenograft models of GBM [9], and even patient-derived xenograft models [10]. However, there have been few clinical reports agreeing that $A T M$ mut $(+)$ could be a prognostic biomarker for a favorable response to RT. Jennifer Ma et al. [23] reported that 8 patients with extracranial primary disease (nonglial tumors) harboring ATM mut(+) demonstrated excellent and durable RT responses with a median local control of 4.62 years; moreover, only 2 patients exhibited local recurrence. Especially, 2 patients with breast and non-small cell lung cancers receiving whole brain RT were observed to relapse at 41.0 and 43.2 months after RT. Additionally, Su et al. [24] reported the significant benefit of adjuvant RT in 43 carriers of germline ATM mutations diagnosed with breast cancer; they reported that 13 patients treated with RT (not cobalt therapy) remained locally diseasefree, sustaining tolerable toxicities. Recently, we reported that somatic ATM mutations in solid tumors (except brain tumors) were related to markedly improved responses after RT compared with tumors not harboring ATM mutations: an overall response rate of $61.0 \%$ (vs. $24.0 \%$ in $A T M$ mut(-) tumors) with a durable response for a median period of 11 mo (vs. 3 mo in ATM mut(-)) [25]. In the current study, the ATM mut(+) group was shown to yield excellent in-field control, indicating the radio-sensitive nature of $A T M$ mutations even in patients with intracranial high-grade glioma.

Following the generation of radiation-induced doublestrand breaks in the DNA, the ATM gene is known to control the key signaling pathway in the repair process of the DNA double-strand break damage [3, 26]. After it has been recruited to the site of the double-strand break by the MRE11-RAD50-NBS1 complex, ATM is activated by autophosphorylation at Ser1981 [27]. Catalytic activation of $A T M$ is known to subsequently lead to the phosphorylation of many downstream effectors involved in the activation of the G1/S cell-cycle checkpoint arrest (CHk1 and Chk2) [28], homologous recombination or non-homologous end-joining repair of DNA [29, 30], and apoptosis (p53) [31]. Consequently, mutations in ATM might prevent the repair or restoration of radiation-induced damaged DNA, resulting in the radiosensitivity of cells. Therefore, germline ATM mut(+) is highly involved in radiation-induced risks from its radiosensitivity. Recently, there is an effort to predict individual response to radiation based on ATM nucleoshuttling rate [32]. Future investigations to increase the understanding of ATM is needed for refinement and development models of personalized ionizing radiation response.

From a clinical point of view, targeting $A T M$ has intrigued many researchers and encouraged them to attempt approaches to enhance the radio-sensitivity of cells. Since then, several ATM inhibitors have been developed and their in vitro or in vivo radio-sensitivity 
Table 3 Prognostic factors for in-field, out-field control, and overall survival determined using univariable Cox regression analysis

\begin{tabular}{|c|c|c|c|}
\hline In-field control & $\mathrm{HR}$ & $95 \% \mathrm{Cl}$ & $P$-value \\
\hline ATM (mut(-) vs. mut(+)) & 0.16 & $0.00-0.48$ & 0.036 \\
\hline Age at diagnosis (<60 vs. $\geq 60$ ) & 1.02 & $0.39-2.69$ & 0.966 \\
\hline Sex (male vs. female) & 1.09 & $0.45-2.78$ & 0.857 \\
\hline KPS (90-100 vs. $\leq 80)$ & 1.44 & $0.51-4.07$ & 0.496 \\
\hline SVZ (free vs. involvement) & 0.97 & $0.28-3.39$ & 0.974 \\
\hline Gliomatosis (No vs. Yes) & 1.34 & $0.53-3.39$ & 0.534 \\
\hline Extent of resection (subtotal vs. partial/biopsy) & 1.15 & $0.45-2.94$ & 0.767 \\
\hline Pathology (WHO Grade II-III vs. WHO grade IV) & 1.47 & $0.34-6.43$ & 0.606 \\
\hline MGMT promoter (unmethylated vs methylated) & 0.59 & $0.23-1.48$ & 0.260 \\
\hline Ki67 index (< 15\% vs. $\geq 15 \%)$ & 1.57 & $0.57-4.32$ & 0.380 \\
\hline BRCA status (wild-type vs mutant) & 1.46 & $0.51-4.19$ & 0.485 \\
\hline PTEN status (wild-type vs mutant) & 1.32 & $0.47-3.72$ & 0.600 \\
\hline TERT status (wild-type vs mutant) & 0.93 & $0.37-2.35$ & 0.873 \\
\hline EGFR amplification (No vs. Yes) & 1.98 & $0.74-5.34$ & 0.176 \\
\hline TP53 status (wild-type vs mutant) & 1.39 & $0.48-4.01$ & 0.546 \\
\hline Out-field control & $H R$ & $95 \% \mathrm{Cl}$ & P-value \\
\hline ATM (mut(-) vs. mut(+)) & 0.92 & $0.35-2.40$ & 0.862 \\
\hline Age at diagnosis (<60 vs. $\geq 60$ ) & 1.58 & $0.68-3.71$ & 0.290 \\
\hline Sex (male vs. female) & 0.95 & $0.40-2.26$ & 0.909 \\
\hline KPS (90-100 vs. $\leq 80)$ & 1.36 & $0.55-3.39$ & 0.506 \\
\hline SVZ (free vs. involvement) & 1.53 & $0.45-5.22$ & 0.496 \\
\hline Gliomatosis (No vs. Yes) & 2.32 & $0.96-5.61$ & 0.062 \\
\hline Extent of resection (subtotal vs. partial/biopsy) & 0.74 & $0.32-1.74$ & 0.497 \\
\hline Pathology (WHO Grade II-III vs. WHO grade IV) & 2.62 & $0.93-7.38$ & 0.069 \\
\hline MGMT promoter (unmethylated vs methylated) & 0.49 & $0.21-1.14$ & 0.098 \\
\hline Ki67 index (<15\% vs. $\geq 15 \%)$ & 1.35 & $0.55-3.33$ & 0.511 \\
\hline BRCA status (wild-type vs mutant) & 2.44 & $1.18-6.07$ & 0.035 \\
\hline PTEN status (wild-type vs mutant) & 0.96 & $0.35-2.62$ & 0.939 \\
\hline TERT status (wild-type vs mutant) & 0.48 & $0.20-1.14$ & 0.096 \\
\hline EGFR amplification (No vs. Yes) & 3.09 & $1.09-8.77$ & 0.035 \\
\hline TP53 status (wild-type vs mutant) & 1.80 & $0.67-4.83$ & 0.246 \\
\hline Overall survival & $H R$ & $95 \% \mathrm{Cl}$ & P-value \\
\hline ATM (mut(-) vs. mut(+)) & 0.77 & $0.27-2.15$ & 0.615 \\
\hline Age at diagnosis (<60 vs. $\geq 60$ ) & 2.27 & $0.89-5.84$ & 0.088 \\
\hline Sex (male vs. female) & 1.01 & $0.40-2.54$ & 0.986 \\
\hline KPS (90-100 vs. $\leq 80)$ & 1.96 & $0.69-5.57$ & 0.205 \\
\hline SVZ (free vs. involvement) & 1.55 & $0.36-6.79$ & 0.559 \\
\hline Gliomatosis (No vs. Yes) & 1.74 & $0.68-4.46$ & 0.246 \\
\hline Extent of resection (subtotal vs. partial/biopsy) & 0.74 & $0.30-1.84$ & 0.518 \\
\hline Pathology (WHO Grade II-III vs. WHO grade IV) & 0.47 & $0.16-1.43$ & 0.184 \\
\hline MGMT promoter (unmethylated vs methylated) & 1.04 & $0.40-2.72$ & 0.931 \\
\hline Ki67 index (<15\% vs. $\geq 15 \%)$ & 1.22 & $0.48-3.10$ & 0.678 \\
\hline
\end{tabular}


Table 3 Prognostic factors for in-field, out-field control, and overall survival determined using univariable Cox regression analysis (Continued)

\begin{tabular}{llll}
\hline BRCA status (wild-type vs mutant) & 2.78 & $1.05-7.36$ & 0.039 \\
PTEN status (wild-type vs mutant) & 1.00 & $0.33-3.04$ & 0.992 \\
TERT status (wild-type vs mutant) & 0.88 & $0.36-2.18$ & 0.785 \\
EGFR amplification (No vs. Yes) & 1.78 & $0.67-4.72$ & 0.249 \\
TP53 status (wild-type vs mutant) & 0.42 & $0.10-1.80$ & 0.241 \\
\hline
\end{tabular}

*The foreparts of the parentheses were set as the reference group

Abbreviations: HR hazards ratio, Cl confidence interval, KPS Karnofsky performance status, SVZ subventricular zone, MGMT

O[6]-methylguanine-DNA methyltransferase

have been reported [17]. However, to date, no ATM inhibitor has yet been clinically used because of the lack of bioavailability, concerns of potential side-effects, and the lack of clinical trials. There is an ongoing prospective phase I trial on the combination of palliative RT and an ATM inhibitor against solid tumors (NCT03225105). In addition to the administration of ATM inhibitors as radio-sensitizers, somatic $A T M$ mutations could also be applied as intrinsic radio-sensitizers. The advent of NGS in routine clinical practice might allow ATM to be used as the sole biomarker for patients treated with RT. Recently, researchers developed a genome-adjusted radiation dose model based on an individualized radiosensitivity index with genomic features [33]. Beyond personalized radiation dose planning, we could cautiously assume that whole brain or large field RT rather than focal RT might be an effective personalized treatment option with excellent in-field control and prolonged survival in patients with IDH-wildtype ATM mut(+) highgrade glioma.

In addition, the current study showed that $B R C A$ mutations could be associated with poor outcomes of not only intracranial control but also OS. Our results appeared to differ to some extent from those demonstrated on different primary tumors, many of which have reported the survival benefit of $B R C A$ mutations. To date, there has not been any clinical evidence supporting the notion that a somatic BRCA mutation might govern the prognosis of IDH-wildtype high-grade glioma. Recent studies of germline BRCA1/2 mutations in patients with breast cancer have suggested that these mutations have a similar prognosis, though they exhibit different effects on the efficacy of chemotherapy [34, 35]. Additionally, there have been several reports analyzing the survival benefit of $B R C A 1 / 2$ mutations in patients with ovarian cancer [36]. These data have collectively supported the relatively better short-term prognosis in patients carrying $B R C A 1 / 2$ mutations. In the current study, a subgroup analysis stratified by the mutational status of both the BRCA and ATM genes revealed that patients without any mutation showed comparable outcomes to the historical data, whereas the ATM mut(-)/BRCA mut(+) group showed the worst outcomes. The ATM mut(+) group with or without $B R C A$ mutations showed comparable results of rates of out-field control and OS, with the same excellent in-field control. These results reflected the fact that $B R C A$ might be a target in the downstream signaling of $A T M$. Further investigation regarding the prognostic impact of $B R C A$ on high-grade glioma is needed.

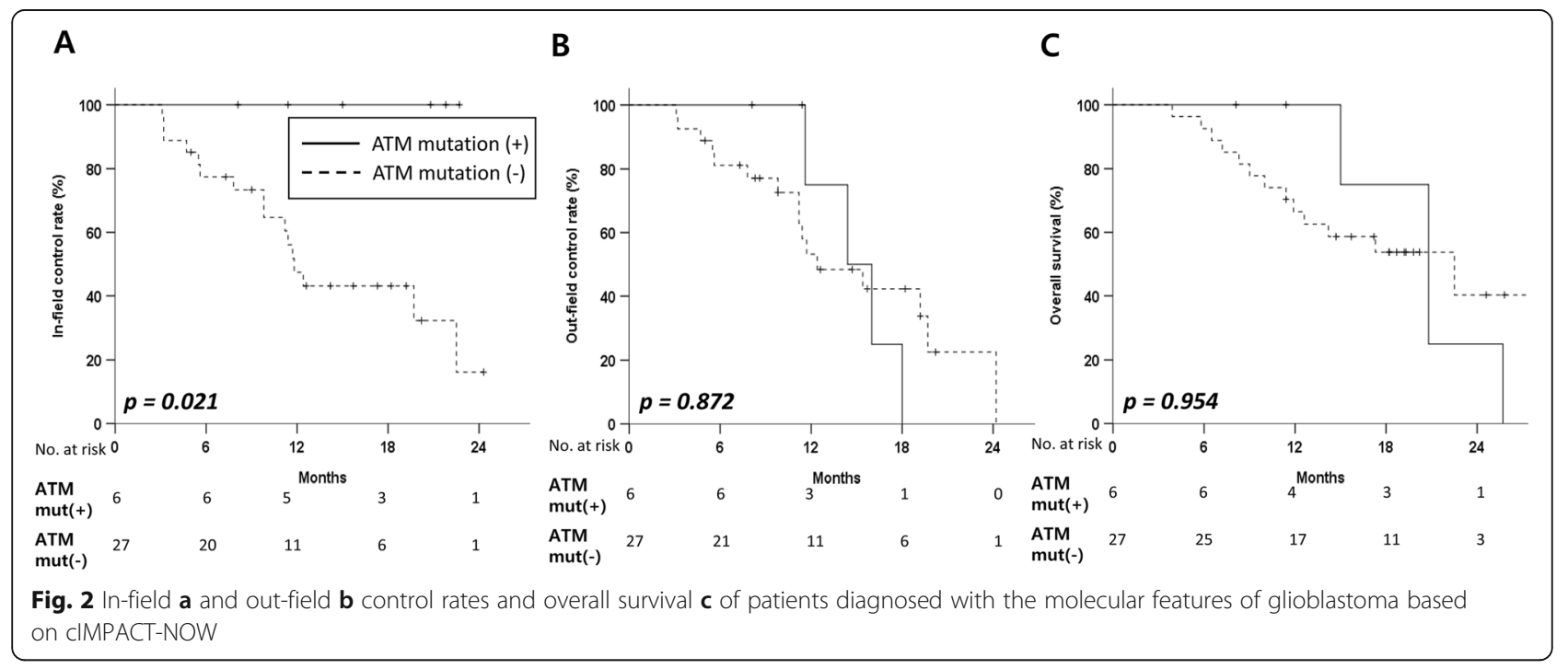




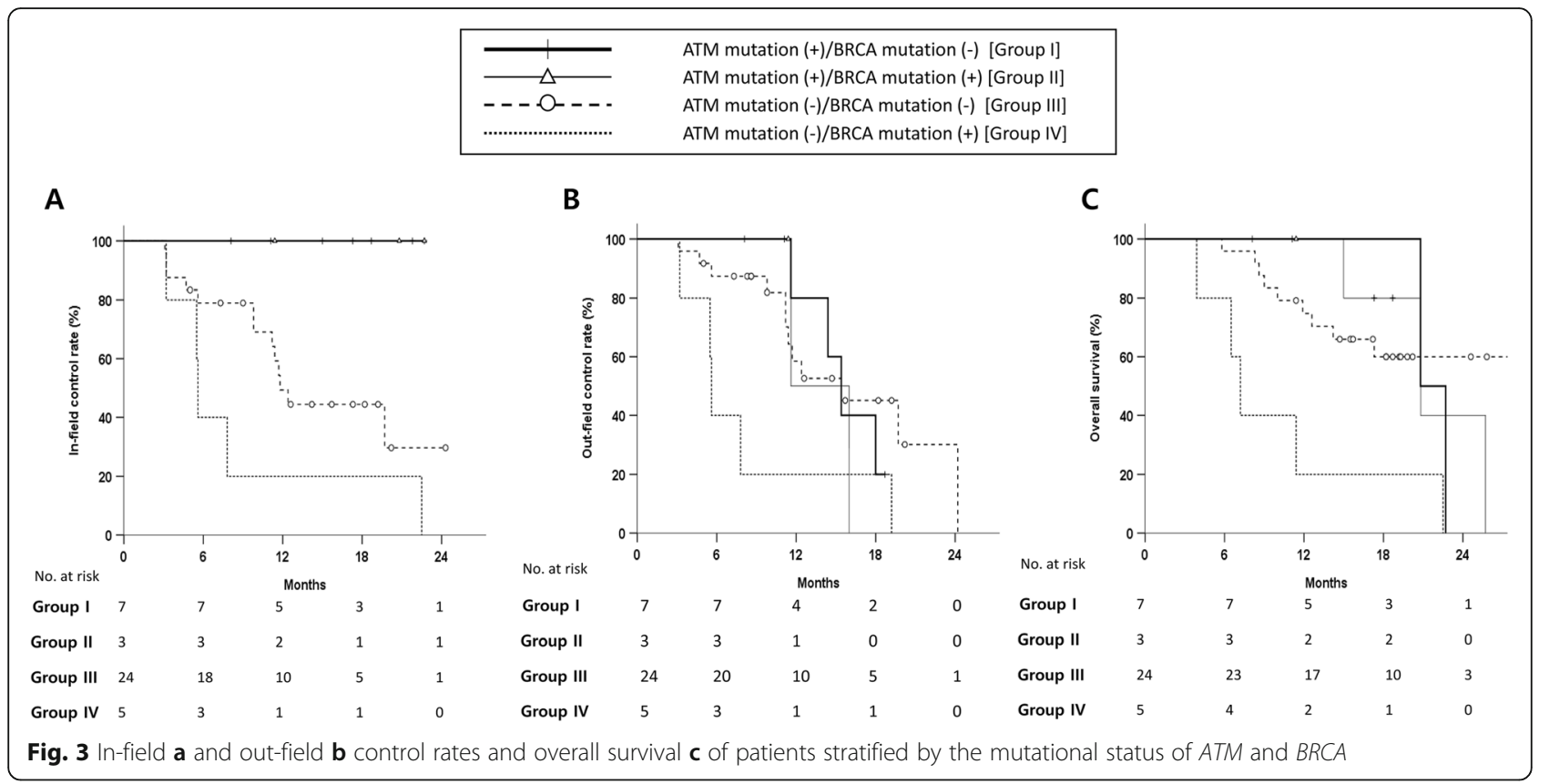

Our study had several limitations. First, this was a retrospective analysis; thus, the results should be cautiously reviewed. Second, owing to the limited number of patients, there are issues regarding the statistical insignificance of the results and the unavailability of multivariable analysis to minimize potential confounders. However, to our knowledge, this study has the largest clinical data, to date, on tumors with somatic ATM mutations analyzed by the latest NGS technology. In addition, a relatively frequent $A T M \operatorname{mut}(+)$ in the current cohort compared to previous report could overestimate the potential predictive value of ATM mut(+); however, the widespread utilization of NGS is expected to clarify the real-world prevalence of ATM mut(+). An additional limitation was that this was a single-center study; however, its consistent treatment strategy of surgery and RT provides another strength to this study. Lastly, the in-field control rates of the ATM mut(+) group could have been overestimated owing to the short-term follow-up of this study. As most patients with IDH-wildtype high-grade glioma were observed to experience in-field or out-field failures within 1 year after diagnosis, the current follow-up period was considered acceptably reasonable for the analysis of failure patterns. However, long-term follow-up data based on a larger number of patients is still needed to efficiently determine certain effects of $A T M$ mutations.

\section{Conclusions}

We demonstrated that ATM mutations in IDH-wildtype high-grade glioma could yield an excellent RT response, irrespective of the adverse features of gliomatosis or the involvement of the SVZ. Owing to the limited number of ATM mutations in high-grade glioma, a further validation in a larger cohort, even including patients under gross total removal status, is needed to identify the prognostic value of ATM mutations. Therefore, we have initiated a multicenter cohort study with Korean Radiation Oncology Group (Protocol No. KROG 19-11). Further preclinical and prospective clinical studies are warranted to elucidate the role of large field RT in patients with $A T M$ mut(+) and the effect of the combination of RT and ATM inhibitors during the course of RT in IDHwildtype high-grade glioma.

\section{Supplementary information}

Supplementary information accompanies this paper at https://doi.org/10. 1186/s13014-020-01619-y.

Additional file 1. Mutations in other genes according to the mutational status of ATM.)

Additional file 2. Clinical outcomes according to the mutational status of ATM in tumors with the molecular features of glioblastoma based on CIMPACT-NOW.

Additional file 3. In-field (a) and out-field (b) control rates and overall survival (c) of patients presenting SVZ involvement.

\section{Abbreviations}

IDH: Isocitrate dehydrogenase; RT: Radiation therapy; MGMT: O-6methylguanine-DNA methyltransferase; SVZ: Subventricular zone; ATM: Ataxia-telangiectasia mutated; NGS: Next-generation sequencing; IQR: Interquartile range; MRI: Magnetic resonance image; CIMPACTNOW: Consortium to Inform Molecular and Practical Approaches to CNS Tumor Taxonomy; BRCA1/2: Breast Cancer susceptibility gene1/2; OS: Overall survival; KPS: Karnofsky performance scale; GBM: Glioblastoma multiforme 


\section{Acknowledgements}

The results were presented at the 61st annual meeting of American Society for Radiation Oncology, October 2019, Chicago, IL, USA and 2019 Annual meeting of Society of Neuro-Oncology, November 2019, Phoenix, AZ, USA.

\section{Authors' contributions}

Conceptualization: Hong In Yoon, Jong Hee Chang; Methodology: Nalee Kim, Se Hoon Kim, Hong In Yoon; Formal analysis and investigation: Nalee Kim, Se Hoon Kim, Seok Gu Kang, Ju Hyung Moon, Jaeho Cho, Chang-Ok Suh; Writing-original draft preparation: Nalee Kim, Hong In Yoon, Jong Hee Chang; Writing - review and editing: Nalee Kim, Chang-Ok Suh, Hong In Yoon, Jong Hee Chang; Funding acquisition: Hong In Yoon; Resources: Se Hoon Kim, Seok Gu Kang, Ju Hyung Moon, Jaeho Cho, Hong In Yoon; Supervision: Hong In Yoon, Jong Hee Chang. The author(s) read and approved the final manuscript.

\section{Funding}

This work was supported by Radiation Technology R\&D program through the National Research Foundation of Korea funded by the Ministry of Science and ICT (NRF-2017R1C1B2010379).

\section{Availability of data and materials}

It is limited due to institutional data protection law and confidentiality of patient data.

\section{Ethics approval and consent to participate}

This study was approved by the Health Institutional Review Boards of Yonsei University Hospital (No.4-2019-0009). The requirement for informed consent was waived because of the retrospective nature of this study.

\section{Consent for publication}

Not applicable.

\section{Competing interests}

The authors declare that the research was conducted in the absence of any commercial or financial relationships that could be construed as a potential conflict of interest.

\section{Author details}

'Department of Radiation Oncology, Yonsei Cancer Center, Severance Hospital, Yonsei University Health System, Yonsei University College of Medicine, 50-1 Yonsei-ro, Seodaemun-gu, Seoul 03722, Republic of Korea. ${ }^{2}$ Department of Radiation Oncology, Samsung Medical Center, Sungkyunkwan University School of Medicine, Seoul, Republic of Korea. ${ }^{3}$ Department of Pathology, Severance Hospital, Yonsei University Health System, Yonsei University College of Medicine, 50-1 Yonsei-ro, Seodaemun-gu, Seoul 03722, Republic of Korea. ${ }^{4}$ Brain Tumor Center, Severance Hospital, Yonsei University Health System, Yonsei University College of Medicine, 50-1 Yonsei-ro, Seodaemun-gu, Seoul 03722, Republic of Korea. ${ }^{5}$ Department of Neurosurgery, Severance Hospital, Yonsei University Health System, Yonsei University College of Medicine, 50-1 Yonsei-ro, Seodaemun-gu, Seoul 03722, Republic of Korea. ${ }^{6}$ Department of Radiation Oncology, CHA Bundang Medical Center, CHA University, Gyeonggi-Do, Republic of Korea.

Received: 10 May 2020 Accepted: 13 July 2020

Published online: 31 July 2020

\section{References}

1. Bao S, Wu Q, McLendon RE, et al. Glioma stem cells promote radioresistance by preferential activation of the DNA damage response. Nature. 2006;444(7120):756-60

2. Kim N, Chang JS, Wee CW, et al. Validation and optimization of a webbased nomogram for predicting survival of patients with newly diagnosed glioblastoma. Strahlenther Onkol. 2019;196(1):58-69.

3. Choi M, Kipps T, Kurzrock R. ATM mutations in Cancer: therapeutic implications. Mol Cancer Ther. 2016;15(8):1781-91.

4. Lee JM, Ledermann JA, Kohn EC. PARP inhibitors for BRCA1/2 mutationassociated and BRCA-like malignancies. Ann Oncol. 2014;25(1):32-40.
5. Hickson I, Zhao Y, Richardson CJ, et al. Identification and characterization of a novel and specific inhibitor of the ataxia-telangiectasia mutated kinase ATM. Cancer Res. 2004;64(24):9152-9.

6. Rainey MD, Charlton ME, Stanton RV, et al. Transient inhibition of ATM kinase is sufficient to enhance cellular sensitivity to ionizing radiation. Cancer Res. 2008:68(18):7466-74.

7. Roy K, Wang L, Makrigiorgos GM, et al. Methylation of the ATM promoter in glioma cells alters ionizing radiation sensitivity. Biochem Biophys Res Commun. 2006;344(3):821-6.

8. Golding SE, Rosenberg E, Adams BR, et al. Dynamic inhibition of ATM kinase provides a strategy for glioblastoma multiforme radiosensitization and growth control. Cell Cycle. 2012;11(6):1167-73.

9. Biddlestone-Thorpe L, Sajjad M, Rosenberg E, et al. ATM kinase inhibition preferentially sensitizes p53-mutant glioma to ionizing radiation. Clin Cancer Res. 2013;19(12):3189-200

10. Durant ST, Zheng L, Wang Y, et al. The brain-penetrant clinical ATM inhibitor AZD1390 radiosensitizes and improves survival of preclinical brain tumor models. Sci Adv. 2018;4(6):eaat1719.

11. Meyerson M, Gabriel S, Getz G. Advances in understanding cancer genomes through second-generation sequencing. Nat Rev Genet. 2010;11(10):685-96.

12. Lim DA, Cha S, Mayo MC, et al. Relationship of glioblastoma multiforme to neural stem cell regions predicts invasive and multifocal tumor phenotype. Neuro-Oncology. 2007;9(4):424-9.

13. Roh TH, Park HH, Kang SG, et al. Long-term outcomes of concomitant chemoradiotherapy with temozolomide for newly diagnosed glioblastoma patients: a single-center analysis. Medicine (Baltimore). 2017;96(27):e7422.

14. Choi SH, Kim JW, Chang JS, et al. Impact of including Peritumoral edema in radiotherapy target volume on patterns of failure in Glioblastoma following Temozolomide-based Chemoradiotherapy. Sci Rep. 2017;7:42148.

15. Im JH, Hong JB, Kim SH, et al. Recurrence patterns after maximal surgical resection and postoperative radiotherapy in anaplastic gliomas according to the new 2016 WHO classification. Sci Rep. 2018;8(1):777.

16. Na K, Kim HS, Shim HS, et al. Targeted next-generation sequencing panel (TruSight tumor 170) in diffuse glioma: a single institutional experience of 135 cases. J Neuro-Oncol. 2019;142(3):445-54.

17. Weber AM, Ryan AJ. ATM and ATR as therapeutic targets in cancer. Pharmacol Ther. 2015;149:124-38.

18. Kim YS, Kim SH, Cho J, et al. MGMT gene promoter methylation as a potent prognostic factor in glioblastoma treated with temozolomide-based chemoradiotherapy: a single-institution study. Int J Radiat Oncol Biol Phys. 2012;84(3):661-7.

19. Brat DJ, Aldape K, Colman H, et al. cIMPACT-NOW update 3: recommended diagnostic criteria for "diffuse astrocytic glioma, IDH-wildtype, with molecular features of glioblastoma, WHO grade IV". Acta Neuropathol. 2018; 136(5):805-10

20. Chukwueke UN, Wen PY. Use of the Response Assessment in NeuroOncology (RANO) criteria in clinical trials and clinical practice. CNS Oncol. 2019;8(1):CNS28-CNS.

21. Taylor AM, Harnden DG, Arlett CF, et al. Ataxia telangiectasia: a human mutation with abnormal radiation sensitivity. Nature. 1975;258(5534):427-9.

22. Chen $\mathrm{PC}$, Lavin MF, Kidson $\mathrm{C}$, et al. Identification of ataxia telangiectasia heterozygotes, a cancer prone population. Nature. 1978;274(5670):484-6.

23. Ma J, Setton J, Morris $L$, et al. Genomic analysis of exceptional responders to radiotherapy reveals somatic mutations in ATM. Oncotarget. 2017;8(6):10312-23.

24. Su Y, Swift M. Outcomes of adjuvant radiation therapy for breast cancer in women with ataxia-telangiectasia mutations. JAMA. 2001;286(18):2233-4.

25. Lee JJB, Yang AJ, Chang JS, et al. Genomic analysis reveals somatic mutations of ATM gene in DNA repair confer exceptional target lesion response to radiation therapy. J Glob Oncol. 2019;5(suppl):130.

26. Shiloh Y, Ziv Y. The ATM protein kinase: regulating the cellular response to genotoxic stress, and more. Nat Rev Mol Cell Biol. 2013;14(4):197-210.

27. Bakkenist CJ, Kastan MB. DNA damage activates ATM through intermolecular autophosphorylation and dimer dissociation. Nature. 2003; 421(6922):499-506.

28. Matsuoka S, Huang M, Elledge SJ. Linkage of ATM to cell cycle regulation by the Chk2 protein kinase. Science. 1998;282(5395):1893-7.

29. Durant ST, Nickoloff JA. Good timing in the cell cycle for precise DNA repair by BRCA1. Cell Cycle. 2005:4(9):1216-22.

30. Durant ST, Paffett KS, Shrivastav M, et al. UV radiation induces delayed hyperrecombination associated with hypermutation in human cells. Mol Cell Biol. 2006;26(16):6047-55. 
31. Blasius M, Bartek J. ATM targets hnRNPK to control p53. Cell Cycle. 2013; 12(8):1162-3.

32. Berthel E, Foray N, Ferlazzo ML. The Nucleoshuttling of the ATM Protein: A Unified Model to Describe the Individual Response to High- and Low-Dose of Radiation? Cancers (Basel). 2019;11(7).

33. Scott JG, Berglund A, Schell MJ, et al. A genome-based model for adjusting radiotherapy dose (GARD): a retrospective, cohort-based study. Lancet Oncol. 2017;18(2):202-11.

34. Hahnen E, Lederer B, Hauke J, et al. Germline mutation status, pathological complete response, and disease-free survival in triple-negative breast Cancer: secondary analysis of the GeparSixto randomized clinical trial. JAMA Oncol. 2017;3(10):1378-85.

35. Copson ER, Maishman TC, Tapper WJ, et al. Germline BRCA mutation and outcome in young-onset breast cancer (POSH): a prospective cohort study. Lancet Oncol. 2018;19(2):169-80.

36. McLaughlin JR, Rosen B, Moody J, et al. Long-term ovarian cancer survival associated with mutation in BRCA1 or BRCA2. J Natl Cancer Inst. 2013; 105(2):141-8.

\section{Publisher's Note}

Springer Nature remains neutral with regard to jurisdictional claims in published maps and institutional affiliations.

Ready to submit your research? Choose BMC and benefit from:

- fast, convenient online submission

- thorough peer review by experienced researchers in your field

- rapid publication on acceptance

- support for research data, including large and complex data types

- gold Open Access which fosters wider collaboration and increased citations

- maximum visibility for your research: over $100 \mathrm{M}$ website views per year

At $\mathrm{BMC}$, research is always in progress.

Learn more biomedcentral.com/submissions 